\title{
Competencias comunicativas comportamentales: Propuesta de una matriz de observación para estudiantes de pedagogía en contexto de diversidad cultural
}

\author{
Communicative Behavioral Competences: Proposal of an Observation Matrix for Pedagogy \\ Students in Context of Cultural Diversity
}

\section{Competências comunicativas comportamentais: Proposta de uma matriz de observação para estudantes de pedagogia no contexto da diversidade cultural}

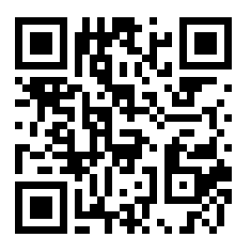

Alejandra Soledad Tapia-Vidal Universidad Católica de Temuco

Temuco, Chile

atapiavidal@gmail.com

https://orcid.org/0000-0002-5391-4354

\begin{abstract}
Recibido • Received • Recebido: 08 / 08 / 2018
Corregido • Revised • Revisado: 02 / 08 / 2019

Aceptado • Accepted • Aprovado: 29/ $10 / 2019$
\end{abstract}

\begin{abstract}
Resumen: Las competencias comunicativas interculturales han sido analizadas profusamente durante 15 años; sin embargo, las herramientas desarrolladas para evaluar han sido autorreportes sobre dimensiones acotadas en competencias comunicativas afectivas y cognitivas. No obstante, la dimensión sobre competencias comunicativas comportamentales (CCC desde ahora en adelante) no han recibido especial atención, lo que se ha traducido en un vacío en el desarrollo de esta área. El objetivo de esta investigación es presentar la fiabilidad de un instrumento que permita describir y analizar las CCC de las personas practicantes de pedagogía en contexto de diversidad cultural desde el modelo teórico de Chen y Starosta (1996). Para el desarrollo de la metodología se diseñó un instrumento mediante un análisis teórico de las CCC. A su vez, se desarrolló un proceso de fiabilidad externa a través de juicio de personal experto, un análisis de fiabilidad interna mediante la aplicación de una matriz por sujetos interobservadores y se aplicó el instrumento en contexto real. Los resultados dan cuenta de un buen soporte teórico que sostiene las dimensiones comportamentales en relación con el uso de la voz, kinésica-gestualidad, proxémica y uso del espacio. Asimismo, se revelan indicadores aportados por el equipo experto que contribuyó para ajustar la matriz. De esta manera, el instrumento generado permite, a la persona investigadora, observar, registrar descriptivamente situaciones relacionadas con el lenguaje no verbal y paraverbal del sujeto participante desde un enfoque etnográfico. Finalmente, se analizaron las limitaciones y proyecciones del instrumento para explorar esta dimensión como primer insumo y desafío a profesionales de investigación.
\end{abstract}

Palabras claves: Competencias comunicativas comportamentales; observación participante; fiabilidad. 
doi: http://doi.org/10.15359/ree.24-1.9

URL: http://www.una.ac.cr/educare

CORREO: educare@una.cr

Abstract: Intercultural communicative competences have been analyzed profusely for 15 years, however, the greatest of the tools developed to evaluate have been self-reports on dimensions limited in affective and cognitive communication skills; nevertheless, the dimension on communicative behavioral competences (CCC from now on) has not received special attention, which has translated into a vacuum in the development of this area. The objective of this research is to present the reliability of an instrument that allows describing and analyzing the CCC of pedagogy practitioners in a cultural diversity context the theoretical model of Chen and Starosta (1996). For the development of the methodology, an instrument was designed through a theoretical analysis of CCC. In turn, an external reliability process was developed through expert judgment, an internal reliability analysis through the application of a matrix by interobservers and the instrument was applied in real context. The results show a good theoretical support that supports the behavioral dimensions in relation to the use of the voice, kinésica-gestualidad, proxemic and use of space. Likewise, indicators contributed by the interjueces that contributed to adjust the matrix are revealed. In this way, the generated instrument allows the researcher to observe, record descriptively, situations related to the nonverbal and paraverbal language of the participant from an ethnographic approach. Finally, the limitations and projections of the instrument were analyzed to explore this dimension as a first input and challenge to field researchers.

Keywords: Communicative competencies; participant observation; reliability.

Resumo: As habilidades de comunicação interculturais foram testados extensivamente durante 15 anos, no entanto, a maioria das ferramentas foram desenvolvidas para avaliar foram auto relatos sobre dimensões delimitadas em habilidades comunicativas, afetivas e cognitivas; No entanto, a dimensão da capacidade de comunicação comportamentais (CCC a partir de agora) não têm recebido atenção especial, o que resultou em uma lacuna no desenvolvimento desta área. O objetivo desta pesquisa é apresentar a confiabilidade de um instrumento que permite descrever e analisar as CCC de praticantes de pedagogia em um contexto de diversidade cultural do modelo teórico de Chen e Starosta (1996). Para o desenvolvimento da metodologia, foi elaborado um instrumento através de uma análise teórica das $\mathrm{CCCl}$. Por sua vez, um processo de confiabilidade externa foi desenvolvido através de julgamento de especialistas, uma análise de confiabilidade interna através da aplicação de uma matriz por interobservadores e o instrumento foi aplicado em contexto real. Os resultados mostram um bom suporte teórico que sustenta as dimensões comportamentais em relação ao uso da voz kinésica- gestos, proxêmico e utilização do espaço. Da mesma forma, se revelam indicadores encontrados pela equipe de expertos que contribuíram para ajustar a matriz. Assim, o instrumento gerado permite ao pesquisador observar e registrar de forma descritiva situações que envolvem linguagem não-verbal e para-verbal da pessoa participante, a partir de uma abordagem etnográfica. Por fim, as limitações e projeções do instrumento foram analisadas para explorar essa dimensão como primeiro insumo e desafio aos pesquisadores.

Palavras-chave: Competências comunicativas; observação participante; confiabilidade. 


\section{Introducción}

Las escuelas de la región de La Araucanía se enmarcan en un escenario multicultural debido a la presencia del pueblo mapuche, de descendientes de grupos colonos y, actualmente, la importante notoria presencia de inmigrantes en dicha región; todo ello ha transformado las aulas escolares en espacios diversos culturalmente para practicantes de pedagogía (Jiménez y Fardella, 2015; Mendoza y Sanhueza, 2016).

En este escenario, el estudiantado de pedagogía que realiza práctica en dichas escuelas se ve desafiado a interactuar desde una perspectiva intercultural con el alumnado de una cultura distinta, lo que requiere del manejo de conocimientos sobre su propia cultura y la de una cultura distinta. En el marco de la interacción intercultural, el estudiantado de pedagogía en práctica también debe ser capaz de regular su emoción en las situaciones de incertidumbre a las cuales se puede enfrentar. Esto va más allá de su dominio idiomático, y hace necesario un buen manejo del lenguaje verbal y no verbal (Hernández y Ávila, 2016; Vilà, 2012). Para ello se requiere que practicantes de pedagogía dominen y regulen las competencias comunicativas comportamentales que les permitirán interactuar de forma directa con el alumnado de una cultura distinta para facilitar el aprendizaje. La competencia comunicativa comportamental hace referencia al dominio y utilización del lenguaje no verbal y paraverbal, esto es, elementos relacionados con el uso de la voz, kinésica-gestualidad, uso del espacio y proxémica, entre otros. En este sentido, para el desarrollo de dicha competencia se hace evidente ingresar al aula donde se desempeña el sujeto practicante, observar y describir el lenguaje no verbal y paraverbal que utiliza en la comunicación con el alumnado (Álvarez, 2012; Vilà 2012).

Los estudios sobre competencias comunicativas en contexto de diversidad cultural han sido ampliamente desarrollados mediante la investigación de las dimensiones cognitivas y afectivas (Bennett 1986; Byram, 1997; Chen y Starosta, 1996; Rubio, 2009; Sanhueza, Pauker, San Martín y Friz, 2012; Vilà, 2014). Ello ha evidenciado que los estudios sobre la dimensión comunicativa comportamental en contexto educativo han sido escasos, y a su vez aparecen en las investigaciones científicas como estudios sobre comunicación no verbal o lenguaje no verbal en el aula escolar o universitaria. Dichas investigaciones han aplicado variadas técnicas e instrumentos para estudiar las CCC.

Entre los estudios realizados en Europa se encuentra el de Suchá (2016), quien utilizó la observación no participante de registros audiovisuales sobre la interacción gestual entre hablantes de distinta cultura, mediante el método estadístico de Bootstrap cuantificó la frecuencia y tipo de gestos de estudiantado francés y checo en formación de negociación empresarial. También Cantillo (2014) utilizó cuestionarios para identificar el uso del espacio y movimientos que utiliza el personal docente universitario en su enseñanza. Este instrumento pretende dar a conocer la percepción y preferencia del personal docente y del estudiantado sobre el uso del lenguaje no verbal en el aula universitaria, los datos fueron codificados mediante el programa AQUAD 6. 
doi: http://doi.org/10.15359/ree.24-1.9

URL: http://www.una.ac.cr/educare

CORREO: educare@una.cr

Variados estudios latinoamericanos dan cuenta de la aplicación de instrumentos propios de la investigación cualitativa. Pinto y Ramírez (2014) utilizaron el diario de vida para diagnosticar las dificultades en la enseñanza del francés y caracterizar el aspecto sensomotriz de alumnado colombiano. Este instrumento permite describir las clases de francés que realiza el profesorado con estudiantes de Colombia para implementar estrategias relacionadas con el lenguaje no verbal como facilitadores en el aprendizaje del francés. De manera similar, Hernández (2011) aplicó cuestionarios dirigidos a estudiantes y entrevistas en profundidad para analizar cómo se relaciona la efectividad de la enseñanza y la comunicación no verbal del personal docente veracruzano desde la percepción del estudiantado universitario. Mediante las entrevistas se analizaron "las percepciones de dos muestras distintas de estudiantes, sobre la efectividad de la enseñanza en función de [la de elementos] ... kinésicos, paralingüísticos y apariencia física ... de los maestros" (Hernández, 2011, p. 72).

Por su parte, Shablico (2012) aplicó una guía de observación participante con escala descriptiva de las expresiones categorizadas en: normal, leve o intensa y un diario de campo se utilizó para analizar el lenguaje no verbal de docentes de ciencias de enseñanza media. De esta manera el instrumento pretende obtener elementos de análisis que permitan identificar la fortaleza de expresiones no verbales para mejorar las prácticas de enseñanza.

En resumen, los instrumentos utilizados en los recientes estudios no consideran las categorías básicas e indicadores que emergen de las teorías sobre el lenguaje no verbal y paraverbal, necesarias para describir las CCC. Además, dichos instrumentos se enfocan mayoritariamente en cuantificar frecuencias de movimientos observados o en dar cuenta sobre las percepciones que manejan los grupos participantes sobre el uso del lenguaje no verbal en las prácticas docentes.

En este escenario, el presente estudio se propone construir y validar una matriz de observación como una forma de abordar este vacío teórico y metodológico que este estudio resolverá. Esta matriz se propone observar y registrar las competencias comunicativas comportamentales del estudiantado de pedagogía en la práctica final. Ello forma parte de la metodología aplicada para el estudio de estilos comunicativos en las prácticas de la formación inicial docente. Así también dicha matriz puede ser aplicada a distintos contextos de interacción en escenarios de diversidad cultural.

En consecuencia, este estudio pretende presentar la fiabilidad de una matriz que permita describir y analizar las competencias comunicativas comportamentales de las personas practicantes de pedagogía en contexto de diversidad cultural, de modo que permita ser utilizado por el estudiantado en práctica intermedia o final, y por las personas supervisoras de práctica. 


\section{Referentes teóricos}

Las competencias comunicativas interculturales constituyen habilidades de interpretación y de relación entre las personas, son procesos de interacción, actitudes como curiosidad y disposición a suspender la incredulidad acerca de otras culturas y creencias (Bryman, 1997). Además, dichas competencias se conforman por el conjunto de habilidades cognitivas, afectivas y comportamentales para manifestar conductas apropiadas y efectivas en un contexto social y cultural determinado, que favorezcan un grado de comunicación suficientemente eficaz (Vilà, 2014).

Chen y Starosta (1996) plantean un modelo teórico sobre tres dimensiones de competencias comunicativas interculturales. Primero, la dimensión cognitiva consiste en el conocimiento que tiene la persona hablante respecto de sí misma y de su interlocutor o interlocutora, la conciencia que debe presentar la persona hablante sobre elementos comunicativos-culturales de la propia cultura y de otras. En un contexto de diversidad social y cultural, el sujeto hablante debe presentar control de incertidumbre, alternancia interpretativa y el conocimiento de las similitudes y diferencias culturales. Segundo, la dimensión afectiva o sensibilidad intercultural, entendida como la integración de habilidades como el respeto, el aprecio, la empatía y la aceptación de otras culturas, así como el rechazo al etnocentrismo y al prejuicio (Sanhueza et al., 2012). En contexto de diversidad social y cultural, el hablante debe controlar la ansiedad en situaciones de incertidumbre y mantener una actitud desprejuiciada, de no discriminación, sino una conducta de empatía frente a personas de distinta cultura.

La tercera dimensión, la comportamental o competencias comunicativas comportamentales (CCC) que hace referencia a la kinésica, proxémica y lenguaje paraverbal. La kinésica que consiste en la utilización que hace el sujeto hablante de gestos faciales como elevar las cejas, sonreír o fruncir el ceño, entre otros. Así también los movimientos corporales, por ejemplo, mover la cabeza, manos, brazos, pies y posturas (Knapp, 1982). La proxémica que es la utilización del uso del espacio en función de las distancias que se establecen entre personas interlocutoras cuando se comunican. Hall (1972) plantea tipos de proxémica interaccional, ejemplos: interacción íntima o de confianza que se evidencia entre 15 a $45 \mathrm{~cm}$ de distancia que utilizan los sujetos interlocutores, interacción personal que se manifiesta entre 46 a $120 \mathrm{~cm}$ de distancia que utilizan las personas. A su vez se propone la interacción social que comprende una distancia de 120 a $360 \mathrm{~cm}$ entre los sujetos interlocutores, y la interacción pública que se observa en el uso de una distancia de más de $360 \mathrm{~cm}$. El lenguaje paraverbal o recursos característicos que utiliza el ser hablante al momento de usar la voz. Esto hace referencia al uso de tonos o de inflexiones al hablar, a las variaciones sobre volumen de la voz para enfatizar ideas y el ritmo o fluidez de la voz en la expresión verbal (Corrales, 2013).

Estudios sobre las competencias comunicativas comportamentales son recientes y la gran mayoría se enfocan en el uso del lenguaje gestual o comunicación no verbal. Suchá (2016) 
doi: http://doi.org/10.15359/ree.24-1.9

URL: http://www.una.ac.cr/educare

CORREO: educare@una.cr

plantea que la cantidad y calidad de las expresiones no verbales durante la comunicación en un idioma extranjero está indudablemente relacionada con la personalidad de quien habla y el nivel de conocimiento de la lengua extranjera. A su vez Cantillo (2014) propone que el uso del lenguaje no verbal por el personal docente universitario tiene implicaciones en la comunicación con el estudiantado, convirtiéndose en una herramienta pedagógica. Así también Pinto y Ramírez (2014) informan que al "aplicar la estrategia con base en la comunicación no verbal se evidenció el mejoramiento de la atención y percepción de los estudiantes en la clase de francés de primer ciclo" (p. 64). Asimismo, Hernández (2011) concluye que el alumnado está atento a los mensajes verbales y no verbales de sus docentes, y clasifican su accionar como bueno o malo para enseñar, por el manejo del contenido, de estrategias pedagógicas y principalmente lo que les rodea como personas.

De esto se infiere que el uso del lenguaje no verbal incide en la práctica pedagógica y se relaciona con la cultura y contexto que acompaña al profesorado. Por otra parte, Shablico (2012) plantea que la gestualidad y movimientos corporales representan recursos expresivos y sensoriales importantes para la enseñanza, también son recursos naturales del profesorado que accede a ellos cada vez que lo necesita. En este sentido el profesorado requiere reflexionar sobre sus capacidades expresivas kinésicas para intencionarlas como estrategia de enseñanza.

Los estudios descritos más arriba evidencian el reciente desarrollo de estudios sobre lenguaje no verbal enfocado más en elementos de la kinésica, como el uso de gestos y movimientos corporales que se utilizan como estrategia para la enseñanza de una lengua extrajera. En consecuencia, se evidencia una escasez de estudios sobre las competencias comunicativas en la dimensión comportamental, enfocándose la mayoría de las investigaciones en indagar sobre las dimensiones cognitiva y afectiva en estudios cualitativos o cuantitativos. Por tanto, desde este escenario de investigación se plantea contribuir con el diseño y proceso de fiabilidad de una matriz de observación de la CCC.

\section{Matriz de observación participante de la CCC}

Metodológicamente, el diseño y fiabilidad del instrumento se enmarca en el proceso de investigación educativa. Esta investigación es definida como la búsqueda sistemática de nuevos conocimientos basada en evidencias sobre los fenómenos para comprender los procesos educativos y mejorar la toma de decisiones en la práctica docente o en la política educativa (Sabariego y Bisquerra, 2014).

El proceso de fiabilidad de la matriz de observación participante sobre las CCC en contexto de diversidad cultural se sustenta en las categorías básicas que emergen de las teorías sobre el lenguaje no verbal y paraverbal expuestas anteriormente en los referentes teóricos, las cuales son fundamentales para la descripción y el análisis de las CCC. Estas categorías dadas por el uso de la voz y el manejo del lenguaje paraverbal, son, por ejemplo: tonos, ritmo, inflexiones y volumen (Corrales, 
2013; Poyatos, 1994); la kinesis-gestualidad, movimientos corporales, posturas y gestos (Knapp, 1982; Poyatos 1994); la proxémica, uso del espacio de acuerdo a las distancias de interacción que mantienen los interlocutores, tales como: íntima, personal, social y pública (Hall, 1972).

En particular, en este estudio se ha utilizado un enfoque etnográfico, lo que implica el interés por la exploración de la naturaleza de un fenómeno social particular que tiende a trabajar primariamente con datos no estructurados. Estos datos no se han codificado en el punto de su recogida, sino fueron analizados mediante la interpretación explícita de los significados y funciones de las acciones humanas, cuyo producto se conforma por descripciones yexplicaciones verbales sobre relaciones sociales, creencias y valores de una comunidad (Angrosino, 2012). A su vez, este enfoque etnográfico aportará para describir los estilos comunicativos que desarrolla el estudiantado de pedagogía con su alumnado en el aula en contexto de diversidad cultural, en relación con la dimensión de la CCC.

\section{Participantes}

Para el proceso de fiabilidad se optó por trabajar con participantes que realizan clases en aula pertenecientes a diversos niveles educativos, en este caso fueron tres profesoras universitarias pertenecientes al programa de bachillerato de una determinada universidad, una docente de lenguaje, una de matemáticas y otra de gestión personal. Asimismo, se incorporaron tres docentes de un liceo: dos profesores de Física y una profesora de Química de octavo año básico. Así también participó una profesora de tercer año básico de una escuela quien aceptó ser registrada audiovisualmente. Por último, se observó una clase de una estudiante en práctica final de una determinada carrera de Pedagogía Básica. La totalidad de participantes firmó la carta de consentimiento informado para resguardar la confidencialidad de la información y su identidad. A su vez se solicitó autorización a los establecimientos donde se desempeñan el grupo participante del proceso de pilotaje de la matriz de observación.

\section{Instrumento}

El instrumento desarrollado se denomina "matriz de observación de competencias comunicativas comportamentales" y se enmarca en la técnica etnográfica de observación participante. Es una técnica entendida como la recogida de datos donde "el investigador ... toma parte [de] su existencia cotidiana y refleja sus interacciones ... en notas de campo que toma en el momento o inmediatamente después de producirse los fenómenos" (Goetz y LeCompte, 1988, p.126). A su vez dicha técnica es definida como "un estilo adoptado por los investigadores de campo que, después de ser aceptados por la comunidad sometida a estudio, pueden utilizar una variedad de instrumentos de recogida de datos para informarse sobre las personas y su modo de vida" (Angrosino, 2012, p. 39). 
doi: http://doi.org/10.15359/ree.24-1.9

URL: http://www.una.ac.cr/educare

CORREO: educare@una.cr

Dicho instrumento tiene como objetivo describir las CCC que desarrollan estudiantes de pedagogía en práctica final.

Las competencias comunicativas comportamentales evaluadas se encuentran dimensionadas con sus respectivas categorías (ver Apéndice A), todas ellas han sido relevadas desde el modelo de Chen y Starosta (1996), los aportes teóricos de Birdwhistell (1970), Hall (1972), Knapp (1982) y Meharabian (1972), entre otros. Fueron ajustadas al contexto de diversidad cultural que caracteriza el aula escolar de la región de la Araucanía.

A continuación, se presentan las categorías e indicadores de la matriz de observación.

a) Uso de la voz, presenta los siguientes indicadores:

1. Varía el tono: consiste en presentar expresividad o inflexiones al hablar.

2. Regula el volumen que puede ser volumen alto, moderado, bajo entre otros; ello de acuerdo con las condiciones del espacio y la cantidad de estudiantes en aula.

3. Varía el ritmo y velocidad de la voz, utiliza pausas según la intención del mensaje.

4. Varía el ritmo y velocidad de la voz, utiliza silencios según la intención del mensaje.

En esta categoría se espera que el estudiantado de pedagogía en práctica dé cuenta de sus habilidades sobre su lenguaje paraverbal, por ejemplo, el uso, regulación o variación de la voz en relación con lo que comunica y enseña mientras interactúa con estudiantes en contexto de diversidad cultural.

b) Kinésica-gestualidad, presenta los siguientes indicadores:

5. Movimientos faciales, movimientos de cejas, ojos, boca u otros.

6. Movimientos corporales, movimientos de cabeza, hombros, manos, pies y posturas.

7. Uso de miradas, contacto visual.

En esta categoría se espera que el estudiantado de pedagogía en práctica exprese sus habilidades en el uso de gestos, movimientos y contacto visual como un complemento estratégico y facilitador del lenguaje verbal que se debe desarrollar al interactuar con sus estudiantes en contexto de diversidad cultural.

c) Proxémica, presenta los siguientes indicadores:

8. Hace uso de las distancias de acuerdo con el mensaje y actividades pedagógicas que se realicen.

9. Hace uso de la cercanía de acuerdo con el mensaje y actividades pedagógicas que se realicen.

10. Atención focalizada individual o hacia un grupo de estudiantes en los casos que se requiera, como una consulta o intervención que pueda realizar. 
En esta categoría se espera que el estudiantado de pedagogía en práctica evidencie sus habilidades en el manejo de la proximidad o lejanía al interactuar con sus estudiantes en contexto de diversidad cultural. A su vez se requiere dar cuenta de cómo el estudiantado en práctica se dispone corporal o posturalmente cuando se aleja o se aproxima a sus estudiantes en el aula.

d) Uso de espacio, se sugiere dibujar en forma sintética:

11. Organización del espacio respecto de la distribución de muebles, pizarra, puerta, ventanas.

12. Trayectoria que utiliza el practicante al ocupar y desplazarse en el espacio.

En esta categoría se espera que el estudiantado de pedagogía en práctica dé cuenta de sus habilidades para usar y organizar el espacio. Esto, en relación con los objetivos de la clase, el contexto de diversidad cultural estudiantil y las condiciones reales que presenta el establecimiento donde se realiza la práctica profesional. Asimismo, se espera evidenciar cómo el estudiantado se relaciona y se apropia del espacio que organiza o distribuye mediante los mismos desplazamientos que realiza.

La matriz presenta inicialmente un registro de datos que contextualizan al sujeto participante observado como: número de caso, género, pedagogía, establecimiento educacional, cantidad de estudiantes, número de estudiantes de distinta cultura, culturas presentes y asignatura. Seguidamente, el instrumento se organiza mediante una tabla que contiene las categorías con sus respectivos indicadores sobre las CCC, el lenguaje no verbal y paraverbal, y, a su vez, el espacio para realizar las observaciones descriptivas según la situación comunicativa o acto pedagógico que desarrolle el estudiantado de pedagogía.

Respecto de la matriz, esta se debe aplicar para observar una determinada clase que desarrolle el estudiantado de pedagogía en práctica final o intermedia. La clase que se observará debe considerar los momentos de toda la clase: inicio, desarrollo y cierre. Esta matriz de observación puede ser aplicada por un sujeto supervisor de prácticas de las carreras de pedagogías, la persona encargada de la unidad técnica pedagógica (UTP) del establecimiento educativo, y una persona investigadora. Además, se sugiere a quienes observan y aplican la matriz ubicarse en un punto fijo que puede ser al final de la sala de clases, según cómo esté organizada la estructura de la sala. Cabe señalar que, si la clase se desarrolla en un espacio abierto, fuera de la sala de clase, el instrumento es igualmente aplicable. Finalmente, se debe registrar en forma secuencial las actividades pedagógicas, y describir en forma acotada los momentos de la clase, estrategias y actividades. Luego, a la misma matriz se le adicionan los indicadores para la interpretación y las conclusiones de las observaciones realizadas. 
doi: http://doi.org/10.15359/ree.24-1.9

URL: http://www.una.ac.cr/educare

CORREO: educare@una.cr

\section{Procedimiento}

El proceso de diseño y de fiabilidad del instrumento se desarrolló en el siguiente orden: el diseño y creación del instrumento mediante revisión de teorías, proceso de fiabilidad externa por juicios experto, proceso de fiabilidad interna por interobservación y la aplicación del instrumento en contexto real. El diseño y creación del instrumento se realizó mediante revisión de teorías sobre las competencias comunicativas interculturales, basándose principalmente en la propuesta de Chen y Starosta (1996), quienes enfatizan la relevancia del uso del lenguaje no verbal y paraverbal en contextos de interculturalidad. Asimismo, se consideraron los aportes sobre la kinésica o lenguaje gestual de Birdwhistell (1970) y los aportes de Meharabian (1972), quien concluye que la comunicación se conforma por un $93 \%$ de lenguaje no verbal y un $7 \%$ de lenguaje verbal. Además, se incorporaron las contribuciones de Hall (1972) en sus estudios sobre el lenguaje de la distancia social denominada proxémica, las bases teóricas de semiótica social de Halliday (1979), y las contribuciones recientes de Knapp (1982) y Poyatos (1994) sobre lenguaje no verbal. Dichas teorías y aportes fueron extraídos en textos de primera fuente y estudios empíricos encontrados en artículos indexados dentro de estos últimos cinco años.

La fiabilidad externa del instrumento "se relaciona con la cuestión de si un investigador independiente descubriría los mismos fenómenos o elaboraría idénticos constructos en el mismo escenario u otro similar" (Goetz y LeCompte, 1988, p. 214). Además, se conforma por factores que dan cuenta de la comparabilidad y traducibilidad de un estudio. La comparabilidad hace referencia al grado en que la definición y descripción de unidades de análisis, participantes, y escenarios del estudio permiten al equipo investigador la comparación de sus resultados con otros estudios relacionados. La traducibilidad consiste en el grado en que el marco teórico, y técnicas de investigación son compresibles para otras investigaciones (Goetz y LeCompte, 1988). Este proceso se realizó mediante el análisis por juicios experto que valoró la calidad, relevancia, comprensión y dimensión de cada ítem. De esta manera, la matriz fue revisada y corregida por cuatro personas académicas relacionadas con el área de la formación inicial docente. Tres profesionales con doctorado en educación; el primero es un académico que ha desarrollado investigaciones sobre educación en contexto de interculturalidad; el segundo, un académico que realiza observaciones a las personas practicantes de pedagogía y, tercero, es una investigadora que ha desarrollado observaciones etnográficas al profesorado en diversos contextos en la región de La Araucanía. Y finalmente, un magíster en educación, quien observa constantemente el desempeño comunicacional del profesorado en el proceso de acompañamiento que realiza en el aula, corrigió el instrumento sobre la base de su trabajo como parte de la unidad técnica pedagógica de un liceo de la región de La Araucanía.

El denominado proceso de fiabilidad interna del instrumento refiere "al grado en que un segundo investigador, a partir de un conjunto de constructos elaborados previamente, ajustaría a ellos sus datos como se hizo en la investigación original" (Goetz y LeCompte, 1988, 
p. 214). Este proceso se desarrolló por tres sujetos interobservadores académicos que aplicaron la matriz propuesta, observaron y registraron información sobre un video que registraba una clase de lenguaje realizada por una profesora de enseñanza básica de tercer año de una escuela municipal de la región de la Araucanía.

Finalmente, se aplicó la matriz en contexto real, se observaron clases realizadas por: tres profesoras universitarias, tres profesores de liceo y una clase realizada por una estudiante de pedagogía en práctica profesional. Ello, para identificar cómo la matriz responde a la observación y registro de las competencias comunicativas comportamentales desarrolladas en los momentos de la clase y en los actos pedagógicos.

\section{Resultados y observaciones tras el proceso de validación}

El objetivo de esta investigación es presentar la fiabilidad de un instrumento 'matriz de observación participante' que permita describir y analizar las CCC de las personas practicantes de pedagogía en contexto diversidad cultural. De modo que pueda ser utilizado por el estudiantado de pedagogía que realice su práctica inicial, intermedia o final y por el personal supervisor de práctica profesional; con la finalidad de contribuir al desarrollo de una óptima, adecuada y pertinente interacción comunicativa con el estudiantado de una cultura distinta, facilitando, así, los aprendizajes. Los resultados refieren que el proceso de revisión teórica sobre lenguaje no verbal y paraverbal permitió crear y diseñar un instrumento que se sustenta en las categorías levantadas como es el uso de voz, kinesis-gestualidad y uso del espacio, en conjunto con la orientación de sus respectivos indicadores.

Respecto del proceso de fiabilidad externa realizada por juicios expertos se hicieron ajustes de información sobre el contexto del establecimiento educacional, por ejemplo, cantidad de estudiantes, número según el género de estudiantes, cantidad de estudiantes de distinta cultura, culturas presentes. Estos datos permiten dar cuenta del contexto de diversidad cultural presente en el aula, lo que caracteriza a la región de la Araucanía que es desde donde se enmarca esta investigación. En este sentido, la información sobre contexto es relevante para el proceso de fiabilidad externa, puesto que es uno de los elementos importantes en el enfoque etnográfico, es decir, "la descripción de los contextos físicos, sociales e interpersonales de la recogida de datos aumenta la replicabilidad de los estudios etnográficos" (Goetz y LeCompte, 1988, p. 219). Además, se adicionó como otra categoría, los "actos pedagógicos" (al final de la matriz), dado que es fundamental registrar el objetivo de la clase, los momentos de la clase y sus respectivas actividades; todos elementos importantes que dan sentido a la observación y descripción de las CCC. A su vez, se realizaron correcciones en el formato, organización de la información y espacios para aplicar y desarrollar la matriz de una manera más adecuada. 
doi: http://doi.org/10.15359/ree.24-1.9

URL: http://www.una.ac.cr/educare

CORREO: educare@una.cr

En cuanto al proceso de fiabilidad interna desarrollada por sujetos interobservadores, se detectó la necesidad de realizar ajustes en las instrucciones para la aplicación de la matriz, pues es necesario explicitar con más detalle cómo se debe aplicar y responder la matriz. Por ejemplo, en la categoría organización espacial se sugirió describir y dibujar el espacio o lugar de la clase, la ubicación de los muebles como una forma de guiar a quien observa y así poder dar cuenta de las condiciones del espacio que influyen, y contextualizan aún más la observación y el registro de las CCC. Por tanto, la explicitación detallada de las instrucciones en la matriz de observación es fundamental, debido a que el proceso de fiabilidad interna pretende que el sujeto interobservador coincida "en la descripción y composición de los acontecimientos y no en la determinación de su frecuencia" (Goetz y LeCompte, 1988, p. 221). Cabe mencionar que, en este proceso de observar el registro audiovisual de una clase y aplicar la matriz al mismo tiempo por sujetos interobservadores, emerge la posibilidad de que la matriz puede utilizarse tanto para observar las CCC de la profesora en su desempeño y la reacción de sus estudiantes en el momento de la interacción comunicativa de la clase. No obstante, esta posibilidad está supeditada solo a la presencia de dos o más personas observadoras en aula directa, lo que se transforma en un desafío y proyección para la investigación, dado que es complejo para un solo investigador o una sola investigadora observar y registrar en aula directa la interacción docente-estudiantes en forma simultánea (Postic, 1996).

Respecto de la aplicación del instrumento en contexto real, la matriz propuesta responde a la observación concreta de las CCC dentro de una práctica pedagógica, debido a que el instrumento permite observar y registrar cada categoría sobre el lenguaje no verbal y paraverbal presente y descrito en la matriz. A su vez, el instrumento presenta instrucciones claras y precisas para ser aplicado, integra espacio necesario en el mismo formato de la matriz para describir o dibujar las observaciones con detalle. Cabe destacar que en la aplicación de la matriz, durante el desarrollo de los momentos de la clase, emerge la necesidad de dibujar la organización espacial del aula (distribución de sillas, mesas, librero) para facilitar el registro de las habilidades en el uso y organización del espacio que presenta el sujeto practicante o docente que se observa. Esto en relación con los objetivos de la clase, el contexto de diversidad cultural del estudiantado en aula y las condiciones reales que presenta el establecimiento donde se realiza la práctica pedagógica; como una forma de comprender de qué manera el uso del espacio incide en el proceso de enseñanza-aprendizaje. Asimismo, surge el requerimiento de consignar y dibujar la trayectoria de desplazamientos de los grupos participantes observados para dar sentido al uso del espacio y, su vez, comprender el uso de la proxémica (proximidades o distancias) que realiza la practicante o docente al interactuar con sus estudiantes.

El instrumento es aplicable en distintos niveles educativos en los que se puede enmarcar la práctica pedagógica, en este caso fue posible observar las CCC de prácticas pedagógicas en el nivel de enseñanza básica, enseñanza media y enseñanza universitaria lo que posibilita 
abordar diversos contextos educativos. Ello se traduce en una proyección del instrumento que no solo se enfoca para observar las CCC de estudiantes de pedagogías que realicen la práctica final o intermedia, en enseñanza básica o media, sino también es posible aplicar el instrumento a profesorado que ejerce su labor pedagógica en distintos centros educativos. No obstante, es necesario señalar que la matriz no es un instrumento que generalice o estandarice sobre las CCC de estudiantes de pedagogía o del profesorado, sino que es un instrumento que está diseñado para describir, registrar, analizar y profundizar sobre las CCC como un aporte para los estudios que se vinculan con la comunicación educativa o comunicación en aula en contexto de diversidad cultural.

Por tanto, al considerar el contexto en el cual se enmarca este estudio, la región de la Araucanía, caracterizada por su diversidad cultural debido a la presencia de la población mapuche, de personas inmigrantes o descendientes de colonos, es posible considerar también la presencia de la diversidad social de las personas, su género, nivel educacional, clase social (García, 2004). Esta diversidad social y cultural actualmente caracteriza los contextos educativos tanto a nivel regional, nacional e internacional. Por consiguiente, la matriz se proyecta como un instrumento que aporta para desarrollar más estudios en contexto de interculturalidad, considerando la presencia histórica y actual de población indígena e inmigrantes en Latinoamérica (Marimán, 2019).

\section{Conclusiones}

El estudio expone las fases de un proceso de fiabilidad de una matriz de observación participante correspondiente a una investigación educativa cualitativa que se enmarca en contexto de escuelas diversas culturalmente en la región de La Araucanía.

El instrumento se creó y diseñó mediante las categorías que emergieron de la revisión de estudios teóricos y metodológicos sobre el lenguaje no verbal y paraverbal que permitieran describir las CCC del estudiantado de pedagogía en práctica final o intermedia.

El diseño de la matriz no es un instrumento que generalice o estandarice sobre las CCC, sino que solo puede ser aplicado para observar, registrar, analizar y profundizar sobre las CCC que presentan estudiantes de pedagogía en la práctica final o intermedia. Así también, para el profesorado que esté ejerciendo en contexto de diversidad social y cultural o en contexto de interculturalidad. Ello, como una contribución a los estudios educativos latinoamericanos sobre CCC, dada la significativa presencia de población indígena e inmigrante en las aulas educativas de Latinoamérica.

Las proyecciones de este estudio refieren a que esta matriz puede ser aplicada en distintos niveles educativos como: enseñanza preescolar, enseñanza básica, enseñanza media, enseñanza 
doi: http://doi.org/10.15359/ree.24-1.9

URL: http://www.una.ac.cr/educare

CORREO: educare@una.cr

universitaria en los que se desenvuelva el estudiantado de pedagogía en situación de práctica como el profesorado que ejerza en aula. Asimismo, el instrumento puede ser aplicado por más de un sujeto observador al mismo tiempo, lo que posibilita la observación y el registro de las CCC del sujeto practicante o docente y la reacción del alumnado en el momento de la interacción comunicativa en la clase.

En consecuencia, la matriz se traduce como un aporte para observar, registrar, explorar, describir y retroalimentar el desempeño de estudiantes de pedagogía en relación con sus CCC desarrolladas en las prácticas intermedias o finales en aulas en contexto de diversidad cultural. A su vez, el instrumento se propone como una contribución que permita ampliar el estudio de la dimensión comportamental de las competencias comunicativas, como una forma de aportar en la reflexión sobre el desarrollo de la CCC en las prácticas pedagógicas y su incidencia en los procesos de enseñanza y aprendizaje en contextos de diversidad cultural.

\section{Referencias}

Álvarez, Q. (2012). La comunicación no verbal en los procesos de enseñanza-aprendizaje: El papel del profesor. Innovación Educativa, 22, 23-37. Recuperado de http://www.usc.es/ revistas/index.php/ie/article/view/725/707

Angrosino, M. (2012). Etnografía y observación participante en la investigación cualitativa. Madrid: Morata.

Bennett, M. (1986). A developmental approach to training for intercultural rensitivity. International Journal of Intercultural Relations, 10(2), 179-196. doi: https://doi.org/10.1016/01471767(86)90005-2

Birdwhistell, R. (1970). Kinesics and context: Essays on body motion communication. Philadelpia: University of Pennsylvania. Doi: https://doi.org/10.9783/9780812201284

Byram, M. (1997). Teaching and assessing intercultural communicative competence. USA: Multilingual Matters.

Cantillo, M. G. (2014). El uso de lenguaje no verbal en la comunicación docente universitaria, implicaciones y efectos en la eficacia comunicativa (Tesis de doctorado), Universidad de Alicante, España. Recuperado de https://rua.ua.es/dspace/bitstream/10045/46195/1/ tesis martha graciela cantillo sanabria.pdf

Chen, G.-M., y Starosta, W. J. (1996). Intercultural communication competence: A synthesis. Annals of the International Communication Association, 19(1), 353-383. doi: https://doi.org $\underline{10.1080 / 23808985.1996 .11678935}$ 
Corrales, M. (2013). Estrategias de comunicación efectiva en el aula (Tesis de maestría). Universidad Pública de Navarra. España. Recuperado de https://academica-e.unavarra.es/ xmlui/handle/2454/8167

Goetz, J. P. y LeCompte, M. D. (1988). Etnografía y diseño cualitativo en investigación educativa. Madrid: Morata.

Hall, E. T. (1972). La dimensión oculta. Argentina: Siglo XXI Editores.

Halliday, M. (1979). El lenguaje como semiótica social. La interpretación social del lenguaje y el significado. México: Fondo de Cultura.

Hernández, A y Ávila, I. M. (2016). La competencia comunicativa intercultural en la educación médica oosgraduada cubana Alteridad. Revista de Educación, 11(2), 192-204. doi: https:// doi.org/10.17163/alt.v11n2.2016.05

Hernández, G. (2011). Miradas docentes... percepciones estudiantiles. Revista Docencia e Investigación, 21, 71-87. Recuperado de https://ruidera.uclm.es/xmlui/bitstream/ handle/10578/8962/Miradas\%20docentes...\%20percepciones\%20estudiantiles. pdf? sequence $=1$ \&isAllowed $=y$

Jiménez, F. y Fardella, C. (2015). Diversidad y rol de la escuela. Discursos del profesorado en contextos educativos multiculturales en clave migratoria. Revista mexicana de investigación educativa, 20(65), 419-441. Recuperado de http://www.scielo.org.mx/scielo. php?script=sci arttext\&pid=S1405-66662015000200005\&lng=es\&tlng=.

Knapp, M. L. (1982). La comunicación no verbal. El cuerpo y el entorno. México: Paidos.

Marimán, P. (2019). Los mapuche antes de la conquista militar chileno-argentina. En ¡Allkütunge,wingka! ¡ka kiñechi! Ensayos sobre historias mapuche (pp. 77-194). Chile: Ediciones Comunidad de Historia Mapuche.

Meharabian, A. (1972). Nonverbal communication. USA: Transaction Publishers.

Mendoza, I. y Sanhueza, S. (2016). La diversidad cautiva en la interculturalidad de la política de educación intercultural chilena. Revista Educadi, 1(2), 83-98. Recuperado de http:// educadi.uct.cl/index.php/educadi/issue/view/127

Pinto, C. E. y Ramírez, L. A. (2014). Mejora de los procesos de enseñanza aprendizaje del francés como lengua extranjera (fle) por medio de la comunicación no verbal en los estudiantes de ciclo I de la institución educativa distrital integral la Candelaria (Tesis de licenciatura). Universidad de la Salle, Bogotá. Recuperada de http://repository.lasalle.edu.co/bitstream/ handle/10185/17253/26082113 2014.pdf?sequence=3\&isAllowed=y 
doi: http://doi.org/10.15359/ree.24-1.9

URL: http://www.una.ac.cr/educare

CORREO: educare@una.cr

Postic, M. (1996). Observación y formación de los profesores. España: Morata.

Poyatos. F. (1994). La comunicación no verbal. Cultura, lenguaje y conversación. Madrid: Itsmo.

Rubio, M. (2009). El desarrollo de la competencia comunicativa intercultural en la formación inicial docente. Estudios pedagógicos, 35(1), 273-286. doi: https://doi.org/10.4067/S0718$\underline{07052009000100017}$

Sabariego, M. y Bisquerra, R. (2014). Fundamentos metodológicos de la investigación educativa. En R. Bisquerra (Coord.), Metodología de la investigación educativa (pp. 38-39). Madrid: La Muralla.

Sanhueza, S., Paukner, F., San Martín, V. y Friz, M. (2012). Dimensiones de la competencia comunicativa intercultural (CCl) y sus implicaciones para la práctica educativa. Folios, 36, 131-151. doi: https://doi.org/10.17227/01234870.36folios131.151

Shablico, S. (2012). La comunicación no verbal en el aula, un análisis en la enseñanza disciplinar. Cuadernos de Investigación Educativa, 3(18), 99-121. Doi: https://doi.org/10.18861/ cied.2012.3.18.2710

Suchá, R. (2016). Vícejazyčnost a gestika ve cvičném obchodním jednání Čechů a Francouzů. Akta Fakulty filozofické Západočeské univerzity v Plzni, 3, 7-33. Recuperado de https://otik. uk.zcu.cz/handle/11025/26203

Vilà, R. (2012). Los aspectos no verbales en la comunicación intercultural. Ra Ximha, 8(2), 223239. Recuperado de http://www.redalyc.org/pdf/461/46123366010.pdf

Vilà, R. (2014). Juventud y comunicación intercultural. Currículo sem Fronteiras, 14(1), 111-122. Recuperado de http://www.curriculosemfronteiras.org/vol14iss1articles/banos.pdf 
doi: http://doi.org/10.15359/ree.24-1.9

URL: http://www.una.ac.cr/educare

CORREO: educare@una.cr

Apéndice A: Matriz de observación participante

\begin{tabular}{|c|c|c|c|c|}
\hline Practicante N. ${ }^{\circ}$ & $\begin{array}{l}\text { Género } \\
\text { Género } M(\text { ) } F(\text { ) }\end{array}$ & Pedagogía en: & & $\begin{array}{l}\text { Establecimiento: } \\
\text { Contexto en la sala } \\
\text { Cantidad de estudiantes: } \\
\text { Género M( ) F ( ) } \\
\text { N. }{ }^{\circ} \text { de estudiantes de distinta cultura: } \\
\text { Culturas presentes: } \\
\text { Asignatura: }\end{array}$ \\
\hline Inicio de la observación & hora & Término de la observación & hora & Fecha: \\
\hline Objetivo & \multicolumn{4}{|c|}{ Describir las competencias comunicativas comportamentales que desarrolla el estudiantado en práctica profesional. } \\
\hline
\end{tabular}

1. Observa la clase y describe el comportamiento del sujeto practicante en su interacción comunicativa con estudiantes, de acuerdo a las categorías que se presentan a continuación.

\begin{tabular}{l|l}
$\begin{array}{l}\text { Categorías de las CCC } \\
\text { (Competencia comunicativa comportamental) Uso del lenguaje no verbal } \\
\text { y paraverbal }\end{array}$ & $\begin{array}{l}\text { Observaciones descriptivas según la situación } \\
\text { comunicativa (Momentos de la clase o actividades } \\
\text { pedagógicas) }\end{array}$
\end{tabular}

\section{Uso de la voz:}

1. Modifica o mantiene el tono (expresividad, inflexiones al hablar)

2. Regulación del volumen.

Modifica o mantiene el ritmo (velocidad y fluidez al hablar)

3. Utilización de pausas

4. Utilización de silencios

\section{Gestualidad:}

5. Presencia de movimientos faciales (gestos que intenciona al comunicar, cejas, boca, ojos, otros)

6. Presencia de movimientos corporales intenciona al comunicar (cabeza, manos, hombros posturas)

\section{Uso de las miradas}

\section{Contacto visual}

\section{Proxémica:}

8. Usa las distancias

9. Usa la cercanía

10. Atención focalizada o atención conjunta en forma individual o grupal pedagógicas) 


\section{doi: http://doi.org/10.15359/ree.24-1.9}

URL: http://www.una.ac.cr/educare

CORREO: educare@una.cr

\begin{tabular}{l|l|}
$\begin{array}{l}\text { Uso del espacio: Se sugiere dibujar el espacio en forma sintética para } \\
\text { graficar distribución de muebles, pizarra, y otros. }\end{array}$ & \\
11. Organización del espacio & \\
\hline $\begin{array}{l}\text { 12. Trayectoria del desplazamiento que realiza el practicante en el aula } \\
\text { Se sugiere dibujar sobre el espacio que se graficó anteriormente }\end{array}$ & \\
\hline $\begin{array}{l}\text { Actos pedagógicos } \\
\text { Registrar en forma secuencial y acotada los momentos de la clase, } \\
\text { estrategias pedagógicas, actividades. }\end{array}$ &
\end{tabular}

2. Relaciona las observaciones realizadas anteriormente con teorías, para interpretar y concluir sobre las categorías de competencias comunicativas comportamentales que desarrolla el sujeto practicante de pedagogía.

\begin{tabular}{|c|c|c|}
\hline $\begin{array}{l}\text { Categorías de las CCC } \\
\text { (Competencia comunicativa comportamental) } \\
\text { Uso del lenguaje no verbal y paraverbal }\end{array}$ & Interpretaciones & Asertos / conclusiones \\
\hline $\begin{array}{l}\text { Uso de la voz: } \\
\text { 1. Modificaciones en el tono }\end{array}$ & & \\
\hline 2. Regulación volumen & & \\
\hline $\begin{array}{l}\text { Modificaciones en el ritmo: } \\
\text { 3. Utilización de pausas }\end{array}$ & & \\
\hline 4. Utilización de silencios & & \\
\hline $\begin{array}{l}\text { Gestualidad: } \\
\text { 5. Presencia movimientos faciales }\end{array}$ & & \\
\hline 6. Presencia movimientos corporales & & \\
\hline $\begin{array}{l}\text { Uso de las miradas } \\
\text { 7. Contacto visual }\end{array}$ & & \\
\hline $\begin{array}{l}\text { Proxémica } \\
\text { 8. Uso de las distancias }\end{array}$ & & \\
\hline 9. Uso de la cercanía & & \\
\hline $\begin{array}{l}\text { 10. Atención focalizada, atención conjunta } \\
\text { en forma individual o grupal }\end{array}$ & & \\
\hline $\begin{array}{l}\text { Uso del espacio: } \\
\text { 11. Organización del espacio }\end{array}$ & & \\
\hline $\begin{array}{l}\text { 12. Trayectoria del desplazamiento que } \\
\text { realiza el sujeto practicante en el aula }\end{array}$ & & \\
\hline
\end{tabular}

